\title{
Initial Stress and Gravity on P-Wave Reflection from Electromagneto-Thermo-Microstretch Medium in the Context of Three-Phase Lag Model
}

\author{
F. S. Bayones, ${ }^{1}$ S. M. Abo-Dahab ${ }^{1},{ }^{2,3}$ A. M. Abd-Alla, ${ }^{4}$ S. H. Elhag, ${ }^{1}$ A. A. Kilany ${ }^{4},{ }^{4}$ \\ and M. Elsagheer ${ }^{4}$ \\ ${ }^{1}$ Department of Mathematics and Statistics, College of Science, Taif University, P.O. Box 11099, Taif 21944, Saudi Arabia \\ ${ }^{2}$ Department of Mathematics, Faculty of Science, South Valley University, Qena 83523, Egypt \\ ${ }^{3}$ Department of Computer Science, Faculty of Computers and Information, Luxor University, Luxor, Egypt \\ ${ }^{4}$ Department of Mathematics, Faculty of Science, Sohag University, Sohag 82524, Egypt
}

Correspondence should be addressed to A. A. Kilany; arabyatef@yahoo.com

Received 20 January 2021; Revised 3 April 2021; Accepted 15 April 2021; Published 4 June 2021

Academic Editor: Ahmed Mostafa Khalil

Copyright $\odot 2021$ F. S. Bayones et al. This is an open access article distributed under the Creative Commons Attribution License, which permits unrestricted use, distribution, and reproduction in any medium, provided the original work is properly cited.

The present paper studied the reflection of thermo-microstretch waves under the generalized thermoelasticity theory which is employed to study the reflection of plane harmonic waves from a semi-infinite elastic solid under the effect of the electromagnetic field, initial stress, and gravity. The formulation is applied under the thermoelasticity theory with three-phase lag, and the reflection coefficient ratio variations with the angle of incidence under different conditions are obtained. Numerical results obtained from the present study are presented graphically and discussed. It is observed that the initial stress, gravitation, and electromagnetic field exert some influence in the thermo-microstretch medium due to reflection of P-waves.

\section{Introduction}

In recent decades, the influences of electromagnetic field, initial stress, gravitation, and thermal field have been much pronounced in diverse fields, especially, engineering, geophysics, geology, acoustics, plasma, and physics because of its utilitarian aspects in these fields. The generalized theories of thermoelasticity, which admit the finite speed of a thermal signal, have been the center of interest of active research. Allam et al. [1] discussed the Green-Lindsay model of reflection of $\mathrm{P}$ and SV waves from the free surface of thermoelastic diffusion, solid under influence of the electromagnetic field, and initial stress. Abo-Dahab and Kilicman [2] illustrated the reflection and transmission of Pand SV-wave phenomena at the interface between solid and liquid media with magnetic field and two thermal relaxation times. Othman and Song [3] developed the reflection of plane waves from an elastic solid half-space under hydrostatic initial stress without energy dissipation. Abd-Alla et al.
[4] studied SV wave's incidence at the interface between solid and liquid media under electromagnetic field and initial stress in the context of three thermoelastic theories. Aboueregal and Abo-Dahab [5] discussed the dual-phase model on magneto-thermoelasticity infinite nonhomogeneous solid having a spherical cavity. Zhou et al. [6] developed the reflection and transmission of plane waves at the interface of pyroelectric bimaterials. Singh [7] illustrated the reflection of $\mathrm{P}$ and SV waves from the free surface of an elastic solid with generalized thermodiffusion. Sinha and Sinha [8-10] studied the reflection of thermoelastic waves at a solid half-space with thermal relaxation. Angel and Achenbach [11] discussed the reflection and transmission of elastic waves by a periodic array of crack. Kilany et al. [12] developed photothermal and void effect of a semiconductor rotational medium based on the Lord-Shulman theory. Quintanilla and Racke [13] studied a note on stability of three-phase lag heat conduction. Kothari et al. [14] developed the fundamental solutions of generalized 
thermoelasticity with three-phase lags. Banik and Kanoria [15] illustrated generalized thermoelastic interaction in a functionally graded isotropic unbounded medium due to varying heat source with three-phase lag effect. Chou and Yang [16] developed two-dimensional dual-phase lag thermal behavior in single-/multilayer structures using the space-time conservation element and solution element (CESE) method. Liu [17] studied numerical analysis of dualphase lag and heat transfer in a layered cylinder with nonlinear interface boundary conditions. Marzougui et al. [18] used a computational analysis of heat transport irreversibility phenomenon in a magnetized porous channel. Abo-Dahab et al. [19] developed MHD Casson nanofluid flow over nonlinearly heated porous medium in the presence of extending surface effect with suction/injection. Zaim et al. [20] used Galerkin finite element analysis of magneto-hydrodynamic natural convection of $\mathrm{Cu}$-water-nanoliquid in a baffled U-shaped enclosure. Mebarek-Oudina et al. [21] illustrated magneto-thermal convection stability in an inclined cylindrical annulus filled with a molten metal. Ezzat and Othman [22] investigated the plane waves in an electromagneto-thermoelastic perfect conductivity medium with two relaxation times.

The objective of the present investigation is to determine the reflection of $\mathrm{P}$ waves from the thermo-electro-magnetomicrostretch medium in the context of three-phase lag model under the effect of rotation, electromagnetic field, and gravity with initial stress. The reflection coefficient ratios of various reflected waves with the angle of incidence have been obtained from the 3PHL model and LS theory. Also, the effect of the electromagnetic field and gravity is discussed numerically and illustrated graphically. The physical quantities are obtained and tested by a numerical study using the parameters of $\mathrm{Cu}$ as a target and presented graphically. The distribution of these quantities is represented graphically in the presence and absence of electromagnetic field, initial stress, and gravity field.

\section{Formulation of the Problem}

The equations of generalized thermo-microstretch in a rectangular coordinate system $(x, y, z)$ with $z$-axis directed in the media are used. Constant magnetic field intensity $H=$ $\left(0, H_{0}, 0\right)$ is taken as the direction of the $y$-axis. We begin our consideration with linearized equations of electrodynamics of slowly moving media.

$$
\begin{aligned}
J & =\operatorname{curl} h-\varepsilon_{0} \dot{E}, \\
\operatorname{curl} E & =-\mu_{0} \dot{h}, \\
E & =-\mu_{0}(\dot{u} \times H), \\
\nabla . h & =0 .
\end{aligned}
$$

The constitutive equation in the presence of gravitational and electromagnetic field is

$$
\begin{gathered}
\sigma_{j i, i}+F_{i}+G_{i}=\rho \ddot{u}_{i}, \\
F_{i}=\mu_{0}(J \times H)_{i}, \\
G_{i}=\rho g\left(\frac{\partial w}{\partial x}, 0,-\frac{\partial u}{\partial x}\right) .
\end{gathered}
$$

From the above equations, we can obtain

$$
\begin{aligned}
E & =\mu_{0} H_{0}(\dot{w}, 0,-\dot{u}), \\
h & =\left(0,-H_{0} e, 0\right), \\
J & =\left(-h_{z}-\varepsilon_{0} \mu_{0} H_{0} \ddot{w}, 0, h_{x}+\varepsilon_{0} \mu_{0} H_{0} \ddot{u}\right) .
\end{aligned}
$$

From equations (3) and (4), we obtain

$F=\left(F_{x}, F_{y}, F_{z}\right)=\left(\mu_{0} H_{0}^{2} e_{x}-\varepsilon_{0} \mu_{0}^{2} H_{0}^{2} \ddot{u}, 0, \mu_{0} H_{0}^{2} e_{z}-\varepsilon_{0} \mu_{0}^{2} H_{0}^{2} \ddot{w}\right)$.

So, the displacement vector $\mathbf{u}$ has the components $u_{x}=u(x, z, t), u_{y}=0$, and $u_{z}=w(x, z, t)$.

$$
\begin{aligned}
\sigma_{i j}= & \left(\lambda_{0} \varphi^{*}-P+\lambda u_{k, k}\right) \delta_{i j}+(\mu+k) u_{j, i}+\mu u_{i, j}-k \varepsilon_{i j k} \varphi_{k} \\
& -\widehat{\gamma} T \delta_{i j}-P \omega_{i j}, \\
m_{i j}= & \alpha \varphi_{k, k} \delta_{i j}+\beta \varphi_{i, j}+\gamma \varphi_{j, i}, \\
\lambda_{i}= & \alpha_{0} \varphi_{i}^{*},
\end{aligned}
$$

where $\omega_{i j}=(1 / 2)\left(u_{j, i}-u_{i, j}\right)$.

In component form, the basic governing equations become

$$
\begin{gathered}
\left(\mu+k-\frac{P}{2}\right)\left(\frac{\partial^{2} u}{\partial x^{2}}+\frac{\partial^{2} u}{\partial z^{2}}\right)+\left(\lambda+\mu+\frac{P}{2}\right)\left(\frac{\partial^{2} u}{\partial x^{2}}+\frac{\partial^{2} w}{\partial x \partial \mathrm{z}}\right)-k \frac{\partial \varphi_{2}}{\partial z}+\lambda_{0} \frac{\partial \varphi^{*}}{\partial x}-\widehat{\gamma} \frac{\partial T}{\partial x}+F_{1}+\rho \mathrm{g} \frac{\partial w}{\partial x}=\rho \frac{\partial^{2} u}{\partial t^{2}} \\
\left(\mu+k-\frac{P}{2}\right)\left(\frac{\partial^{2} w}{\partial x^{2}}+\frac{\partial^{2} w}{\partial z^{2}}\right)+\left(\lambda+\mu+\frac{P}{2}\right)\left(\frac{\partial^{2} u}{\partial x \partial z}+\frac{\partial^{2} w}{\partial z^{2}}\right)+k \frac{\partial \varphi_{2}}{\partial x}+\lambda_{0} \frac{\partial \varphi^{*}}{\partial z}-\widehat{\gamma} \frac{\partial T}{\partial z}+F_{3}-\rho \mathrm{g} \frac{\partial u}{\partial z}=\rho \frac{\partial^{2} w}{\partial t^{2}} \\
(\alpha+\beta+\gamma) \nabla(\nabla \cdot \varphi)-\gamma \nabla \times(\nabla \times \varphi)+k(\nabla \times u)-2 k \varphi=\rho \mathrm{j} \frac{\partial^{2} \varphi}{\partial t^{2}} \\
\alpha_{0} \nabla^{2} \varphi^{*}-\frac{1}{3} \lambda_{1} \varphi^{*}-\frac{1}{3} \lambda_{0}(\nabla \cdot \mathbf{u})+\frac{1}{3} \widehat{\gamma}_{1} T=\frac{3}{2} \rho \mathrm{j} \frac{\partial^{2} \varphi^{*}}{\partial t^{2}}
\end{gathered}
$$




$$
K^{*} \nabla^{2} T+\tau_{\nu}^{*} \nabla^{2} \dot{T}+K \tau_{T} \nabla^{2} \ddot{T}=\left(1+\tau_{q} \frac{\partial}{\partial t}+\frac{\tau_{q}^{2}}{2} \frac{\partial^{2}}{\partial t^{2}}\right)\left[\rho C_{e} \ddot{T}+\widehat{\gamma}_{0} \ddot{e}+\widehat{\gamma}_{1} T_{0} \ddot{\varphi}^{*}\right]
$$

such that $\tau_{v}^{*}=K+K^{*} \tau_{v}, \nabla^{2}=\left(\partial^{2} / \partial x^{2}\right)+\left(\partial^{2} / \partial z^{2}\right)$.

The constitutive relation can be written as

$$
\begin{aligned}
\sigma_{x x} & =(\lambda+2 \mu+k) \frac{\partial u}{\partial x}+\lambda \frac{\partial w}{\partial z}+\lambda_{0} \varphi^{*}-\widehat{\gamma} T-P, \\
\sigma_{z z} & =(\lambda+2 \mu+k) \frac{\partial w}{\partial z}+\lambda \frac{\partial u}{\partial x}+\lambda_{0} \varphi^{*}-\widehat{\gamma} T-P, \\
\sigma_{x z} & =\left(\mu+\frac{P}{2}\right) \frac{\partial u}{\partial z}+\left(\mu+k-\frac{P}{2}\right) \frac{\partial w}{\partial x}+k \varphi_{2}, \\
\sigma_{z x} & =\left(\mu+\frac{P}{2}\right) \frac{\partial w}{\partial x}+\left(\mu+k-\frac{P}{2}\right) \frac{\partial u}{\partial z}-k \varphi_{2}, \\
m_{x y} & =\gamma \frac{\partial \varphi_{2}}{\partial x} \\
m_{z y} & =\gamma \frac{\partial \varphi_{2}}{\partial z} \\
\lambda_{x} & =\alpha_{0} \frac{\partial j^{*}}{\partial \mathrm{x}} \\
\lambda_{z} & =\alpha_{0} \frac{\partial j^{*}}{\partial \mathrm{z}}
\end{aligned}
$$

To facilitate the solution, the following dimensionless quantities are introduced:

$$
\begin{aligned}
x_{i}^{\prime} & =\frac{\omega^{*}}{c_{0}} x_{i}, \\
u_{i}^{\prime} & =\frac{\rho c_{0} \omega^{*}}{\widehat{\gamma} T_{0}} u_{i}, \\
\Theta^{\prime} & =\frac{\widehat{\gamma}}{\rho c_{0}^{2}}\left(T-T_{0}\right), \\
\left\{t^{\prime}, \tau_{T}^{\prime}, \tau_{v}^{\prime}, \tau_{q}^{\prime}\right\} & =\omega^{*}\left\{t, \tau_{T}, \tau_{v}, \tau_{q}\right\}, \\
\sigma_{i j}^{\prime} & =\frac{\sigma_{i j}}{\widehat{\gamma} T_{0}}, \\
m_{\mathrm{ij}}^{\prime} & =\frac{\omega^{*}}{c_{0} \widehat{\gamma}_{0}} m_{\mathrm{ij}}, \\
\lambda_{i}^{\prime} & =\frac{\omega^{*}}{c_{0} \widehat{\gamma}_{0}} \lambda_{i}, \\
\varphi^{\prime *} & =\frac{\rho c_{0}^{2}}{\widehat{\gamma}_{0} \varphi^{*}}, \\
\varphi_{2}^{\prime} & =\frac{\rho c_{0}^{2}}{\widehat{\gamma}_{0} T_{0}}, \\
g^{\prime} & =\frac{g}{c_{0} \omega^{*}}, \\
\omega^{*} & =\frac{\rho C_{e} c_{0}^{2}}{k}, \\
h^{\prime} & =\left(h / H_{0}\right), \text { and } \\
& =(\lambda+2 \mu+k / \rho), \\
& 1,2,3 \\
&
\end{aligned}
$$

Using equation (18), the governing equations (7)-(16) recast in the following form (after suppressing the primes):

$$
\begin{gathered}
\left(\frac{2 \mu+2 k-P}{2 \rho c_{0}^{2}}\right) \nabla^{2} u+\left(\frac{2 \lambda+2 \mu+P}{2 \rho c_{0}^{2}}+R_{H}\right) \frac{\partial e}{\partial x}-\frac{k}{\rho c_{0}^{2}} \frac{\partial \varphi_{2}}{\partial z}+\frac{\lambda_{0}}{\rho c_{0}^{2}} \frac{\partial \varphi^{*}}{\partial x}-\frac{\rho c_{0}^{2}}{\gamma T_{0}} \frac{\partial \Theta}{\partial x}+g \frac{\partial w}{\partial x}=\beta^{2} \frac{\partial^{2} u}{\partial t^{2}} \\
\left(\frac{2 \mu+2 k-P}{2 \rho c_{0}^{2}}\right) \nabla^{2} w+\left(\frac{2 \lambda+2 \mu+P}{2 \rho c_{0}^{2}}+R_{H}\right) \frac{\partial e}{\partial z}+\frac{k}{\rho c_{0}^{2}} \frac{\partial \varphi_{2}}{\partial x}+\frac{\lambda_{0}}{\rho c_{0}^{2}} \frac{\partial \varphi^{*}}{\partial z}-\frac{\rho c_{0}^{2}}{\gamma T_{0}} \frac{\partial \Theta}{\partial z}-g \frac{\partial u}{\partial z}=\beta^{2} \frac{\partial^{2} w}{\partial t^{2}} \\
\left(\frac{C_{1}^{2}}{c_{0}^{2}} \nabla^{2}-\frac{C_{2}^{2}}{\omega^{* 2}}-\frac{\partial^{2}}{\partial t^{2}}\right) \varphi^{*}-\frac{C_{3}^{2}}{\omega^{* 2}} e+a_{0} \omega_{0}^{2} \varphi_{2}+\frac{k c_{0}^{2}}{\gamma \omega^{* 2}}\left(\frac{\partial u}{\partial z}-\frac{\partial w}{\partial x}\right)=\frac{\rho j c_{0}^{2}}{\gamma} \frac{\partial^{2} \varphi_{2}}{\partial t^{2}}, \\
C_{k} \nabla^{2} \Theta+C_{\nu} \nabla^{2} \dot{\Theta}+C_{T} \nabla^{2} \ddot{\Theta}=\left(1+\tau_{q} \frac{\partial}{\partial t}+\frac{\tau_{q}^{2}}{2} \frac{\partial^{2}}{\partial t^{2}}\right)\left[\ddot{\Theta}+\varepsilon_{1} \ddot{e}+\varepsilon_{2} \ddot{\varphi}^{*}\right]
\end{gathered}
$$




$$
\begin{aligned}
\sigma_{\mathrm{xx}} & =u_{x}+a_{1} w_{z}+a_{2} \varphi^{*}-a_{3} \Theta-a_{4}, \\
\sigma_{\mathrm{zz}} & =w_{z}+a_{1} u_{x}+a_{2} \varphi^{*}-a_{3} \Theta-a_{4}, \\
\sigma_{\mathrm{xz}} & =a_{5} u_{z}+a_{6} w_{x}+a_{7} \varphi_{2}, \\
\sigma_{\mathrm{zx}} & =a_{5} w_{x}+a_{6} u_{z}-a_{7} \varphi_{2}, \\
m_{\mathrm{xy}} & =a_{8} \frac{\partial \varphi_{2}}{\partial x} \\
m_{\mathrm{zy}} & =a_{8} \frac{\partial \varphi_{2}}{\partial z} \\
\lambda_{x} & =a_{9} \frac{\partial \varphi^{*}}{\partial x} \\
\lambda_{z} & =a_{9} \frac{\partial \varphi^{*}}{\partial z}
\end{aligned}
$$

where

$$
\begin{aligned}
& R_{H}=\frac{\mu_{0} H_{0}^{2}}{\rho c_{0}^{2}} \\
& \beta^{2}=\left(1+\frac{\varepsilon_{0} \mu_{0}^{2} H_{0}^{2}}{\rho}\right) \\
& \left(C_{1}^{2}, C_{2}^{2}, C_{3}^{2}\right)=\frac{2}{9 \rho \mathrm{j}}\left(3 \alpha_{0}, \lambda_{0}, \lambda_{1}\right), \\
& a_{0}=\frac{2 \rho c_{0}^{4} \widehat{\gamma}_{1}}{9 \mathrm{j} \widehat{\gamma}^{2} T_{0} \omega^{* 2}}, \\
& \left(C_{k}, C_{v}, C_{T}\right)=\frac{1}{\rho c_{0}^{2} C_{e}}\left(\mathrm{~K}^{*}, \tau_{v}^{*}, \mathrm{~K} \tau_{T} \omega^{*}\right), \\
& \varepsilon_{1}=\frac{\widehat{\gamma}^{3} T_{0}}{\rho^{3} c_{0}^{4} C_{e}} \\
& \varepsilon_{2}=\frac{\widehat{\gamma}_{1} \widehat{\gamma}^{2} T_{0}^{2}}{\rho^{3} c_{0}^{4} C_{e}} \\
& a_{0}=\frac{2 \rho c_{0}^{4}}{9 j \widehat{\gamma} T_{0} \omega^{* 2}}, \\
& a_{4}=\left(\frac{P}{\widehat{\gamma} T_{0}}+1\right) \text {, } \\
& \left(a_{1}, a_{2}, a_{5}, a_{6}, a_{7}\right)=\frac{1}{\rho c_{0}^{2}}\left(\lambda, \lambda_{0}, \mu+\frac{P}{2}, \mu+k-\frac{P}{2}, k\right), \\
& \left(a_{8}, a_{9}\right)=\frac{\omega^{* 2}}{\rho c_{0}^{4}}\left(\gamma, \alpha_{0}\right) .
\end{aligned}
$$

$$
\frac{k c_{0}^{2}}{\gamma \omega^{* 2}} \nabla^{2} \Psi+\left[\nabla^{2}-\frac{j \rho c_{0}^{2}}{\gamma} \frac{\partial^{2}}{\partial t^{2}}-\frac{2 k c_{0}^{2}}{\gamma \omega^{* 2}}\right] \varphi_{2}=0
$$$$
\left(\frac{C_{1}^{2}}{c_{0}^{2}} \nabla^{2}-\frac{C_{2}^{2}}{\omega^{* 2}}-\frac{\partial^{2}}{\partial t^{2}}\right) \varphi^{*}-\frac{C_{3}^{2}}{\omega^{* 2}} \nabla^{2} \Phi+a_{0} \Theta=0,
$$$$
C_{k} \nabla^{2} \Theta+C_{v} \nabla^{2} \dot{\Theta}+C_{T} \nabla^{2} \ddot{\Theta}
$$$$
=\left(1+\tau_{q} \frac{\partial}{\partial t}+\frac{\tau_{q}^{2}}{2} \frac{\partial^{2}}{\partial t^{2}}\right)\left[\ddot{\Theta}+\varepsilon_{1} \nabla^{2} \ddot{\Phi}+\varepsilon_{2} \ddot{\varphi}^{*}\right],
$$

where

$$
\begin{aligned}
\left(\zeta_{0}, \zeta_{1}, \zeta_{2}, \zeta_{3}\right) & =\frac{1}{1+R_{H}}\left(\beta^{2}, g, \frac{\rho c_{0}^{2}}{\gamma T_{0}}, \frac{\lambda_{0}}{\rho c_{0}^{2}}\right),\left(\zeta_{4}, \zeta_{5}, \zeta_{6}\right) \\
& =\frac{2 \rho c_{0}^{2}}{2 \mu+2 k-P}\left(g, \beta^{2}, k\right) .
\end{aligned}
$$

\section{Solution of the problem}

The displacement components $u(x, z, t)$ and $w(x, z, t)$ may be written in terms of potential functions $\Phi(x, z, t)$ and $\Psi(x, z, t)$ as

We assume now that the solution of equations (32)-(36) takes the following form: 


$$
\begin{aligned}
\left\{\Phi, \Psi, \Theta, \varphi^{*}, \varphi_{2}\right\}= & \left\{\bar{\Phi}, \bar{\Psi}, \bar{\Theta}, \overline{\varphi^{*}}, \overline{\varphi_{2}}\right\} \exp \\
& \cdot[i \xi(x \sin \theta+z \cos \theta)-i \omega t] .
\end{aligned}
$$
get

Substituting equation (38) into equations (32)-(36), we

$$
\begin{aligned}
{\left[-\xi^{2}+\zeta_{0} \omega^{2}\right] \bar{\Phi}+i \zeta_{1} \xi } & \sin \theta \bar{\Psi}-\zeta_{2} \bar{\Theta}+\zeta_{3} \overline{\varphi^{*}}=0 \\
{\left[\xi^{2}-\omega^{2} \zeta_{5}\right] \bar{\Psi}-\zeta_{6} \overline{\varphi_{2}} } & =-\iota \xi \zeta_{4} \sin \theta \bar{\Phi}
\end{aligned}
$$

$$
\begin{gathered}
-p^{*} \xi^{2} \bar{\Psi}+\left[\xi^{2}-\frac{j \rho c_{0}^{2} \omega^{2}}{\gamma}+\frac{2 k c_{0}^{2}}{\gamma \omega^{* 2}}\right] \overline{\varphi_{2}}=0, \\
\frac{C_{3}^{2}}{\omega^{* 2}} \xi^{2} \bar{\Phi}+a_{0} \bar{\Theta}-\left[\frac{C_{1}^{2}}{c_{0}^{2}} \xi^{2}+\frac{C_{2}^{2}}{\omega^{* 2}}-\omega^{2}\right] \overline{\varphi^{*}}=0, \\
-\varepsilon_{1} \omega^{2} \tau_{q}^{*} \xi^{2} \bar{\Phi}+\left[\left(-C_{k}-i \omega C_{v}+\omega^{2} C_{T}\right) \xi^{2}+\omega^{2} \tau_{q}^{*}\right] \bar{\Theta} \\
+\varepsilon_{2} \omega^{2} \tau_{q}^{*} \overline{\varphi^{*}}=0,
\end{gathered}
$$

where $\tau_{q}^{*}=1-i \omega \tau_{q}-\left(\omega^{2} \tau_{q}^{2} / 2\right), p^{*}=\left(k c_{0}^{2} / \gamma \omega^{* 2}\right)$.

From equations (39)-(43), we get which tends to

$$
A \xi^{10}+B \xi^{8}+C \xi^{6}+D \xi^{4}+E \xi^{2}+F=0
$$

where $A, B, C, D, E$, and $F$ in equation (45) are given in Appendix A
From equation (45), there are five waves with five different velocities.

Then $\Phi, \Psi, \Theta, \varphi^{*}$, and $\varphi_{2}$ will take the following forms:

$$
\begin{aligned}
\left\{\Phi, \Psi, \Theta, \varphi^{*}, \varphi_{2}\right\}= & \left\{1, \eta_{0}, \kappa_{0}, \chi_{0}, \vartheta_{0}\right\} A_{0} \exp \left[i \xi\left(x \sin \theta_{0}+z \cos \theta_{0}\right)-i \omega t\right] \\
& +\sum_{j=1}^{5}\left\{1, \eta_{j}, \kappa_{j}, \chi_{j}, \vartheta_{j}\right\} A_{j} \exp \left[i \xi_{j}\left(x \sin \theta_{j}-z \cos \theta_{j}\right)-i \omega t\right]
\end{aligned}
$$

From equation (40) and (41), we get $\eta_{j}, \kappa_{j}, \chi_{j}$, and $\vartheta_{j}$ which are given in Appendix B.
3.1. The Boundary Conditions. The boundary conditions for the problem be taken as 


$$
\begin{aligned}
& \sigma_{z z}+\tau_{z z}=0, \sigma_{x z}+\tau_{x z}=0, \frac{\partial T}{\partial z}=0, m_{y z}=0, \lambda_{x}=0, \text { at } z=0, \\
& \sum_{j=1}^{5}\left[a_{1} \vartheta_{j}-\mu_{e} H_{0}^{2} \xi_{j}^{2}-a_{3} \xi_{j}^{2}\left(\sin ^{2} \theta_{j}+\frac{\eta_{j}}{2} \sin 2 \theta_{j}\right)-a_{2} \xi_{j}^{2}\left(\cos ^{2} \theta_{j}-\frac{\eta_{j}}{2} \sin 2 \theta_{j}+\frac{k_{j}}{\xi_{j}^{2}}\right)\right] A_{j} \\
& =\left[a_{1} \vartheta_{1}-\mu_{e} H_{0}^{2} \xi_{1}^{2}-a_{3} \xi_{1}^{2}\left(\sin ^{2} \theta_{0}+\frac{\eta_{1}}{2} \sin 2 \theta_{0}\right)-a_{2} \xi_{1}^{2}\left(\cos ^{2} \theta_{0}-\frac{\eta_{1}}{2} \sin 2 \theta_{0}+\frac{k_{1}}{\xi_{1}^{2}}\right)\right] A_{0}, \\
& \sum_{j=1}^{5}\left[\left(a_{4}+a_{5}\right) \frac{\xi_{j}^{2}}{2} \sin 2 \theta_{j}+a_{4} \xi_{j}^{2} \eta_{j} \cos ^{2} \theta_{j}-a_{5} \xi_{j}^{2} \eta_{j} \sin ^{2} \theta_{j}+a_{6} \xi_{j}\right] A_{j} \\
& =\left[-\left(a_{4}+a_{5}\right) \frac{\xi_{1}^{2}}{2} \sin 2 \theta_{0}+a_{4} \xi_{1}^{2} \eta_{1} \cos ^{2} \theta_{0}-a_{5} \xi_{1}^{2} \eta_{1} \sin ^{2} \theta_{0}+c a_{6} \xi_{1}\right] A_{0}, \\
& \\
& \quad \sum_{j=1}^{5} k_{j} \xi_{j} \cos \theta_{j} A_{j}=k_{1} \xi_{1} \cos \theta_{0} A_{0}, \\
& \\
& \sum_{j=1}^{5} \zeta_{j} \xi_{j} \cos \theta_{j} A_{j}=\zeta_{1} \xi_{1} \cos \theta_{0} A_{0}, \\
& \sum_{j=1}^{5} \vartheta_{j} \xi_{j} \cos \theta_{j} A_{j}=-\vartheta_{1} \xi_{1} \cos \theta_{0} A_{0} .
\end{aligned}
$$

From equations (55)-(60), we get

$$
\begin{aligned}
a_{i j} Z_{j} & =B_{i}, \quad i, j=(1,2, \ldots, 5), \\
Z_{j} & =\frac{A_{j}}{A_{0}}, \\
a_{1 j} & =a_{1} \vartheta_{j}-\xi_{j}^{2}\left[a_{3}\left(\sin ^{2} \theta_{j}+\frac{\eta_{j}}{2} \sin 2 \theta_{j}\right)-a_{2}\left(\cos ^{2} \theta_{j}-\frac{\eta_{j}}{2} \sin 2 \theta_{j}+\frac{k_{j}}{\xi_{j}^{2}}\right)+\mu_{e} H_{0}^{2}\right], \\
a_{2 j} & =\left(a_{4}+a_{5}\right) \frac{\xi_{j}^{2}}{2} \sin 2 \theta_{j}+a_{4} \xi_{j}^{2} \eta_{j} \cos ^{2} \theta_{j}-a_{5} \xi_{j}^{2} \eta_{j} \sin ^{2} \theta_{j}+a_{6} \xi_{j}, \\
a_{3 j} & =k_{j} \xi_{j} \cos \theta_{j}, \\
a_{4 j} & =\zeta_{j} \xi_{j} \cos \theta_{j} A_{j}, \\
a_{5 j} & =\vartheta_{j} \xi_{j} \cos \theta_{j},
\end{aligned}
$$




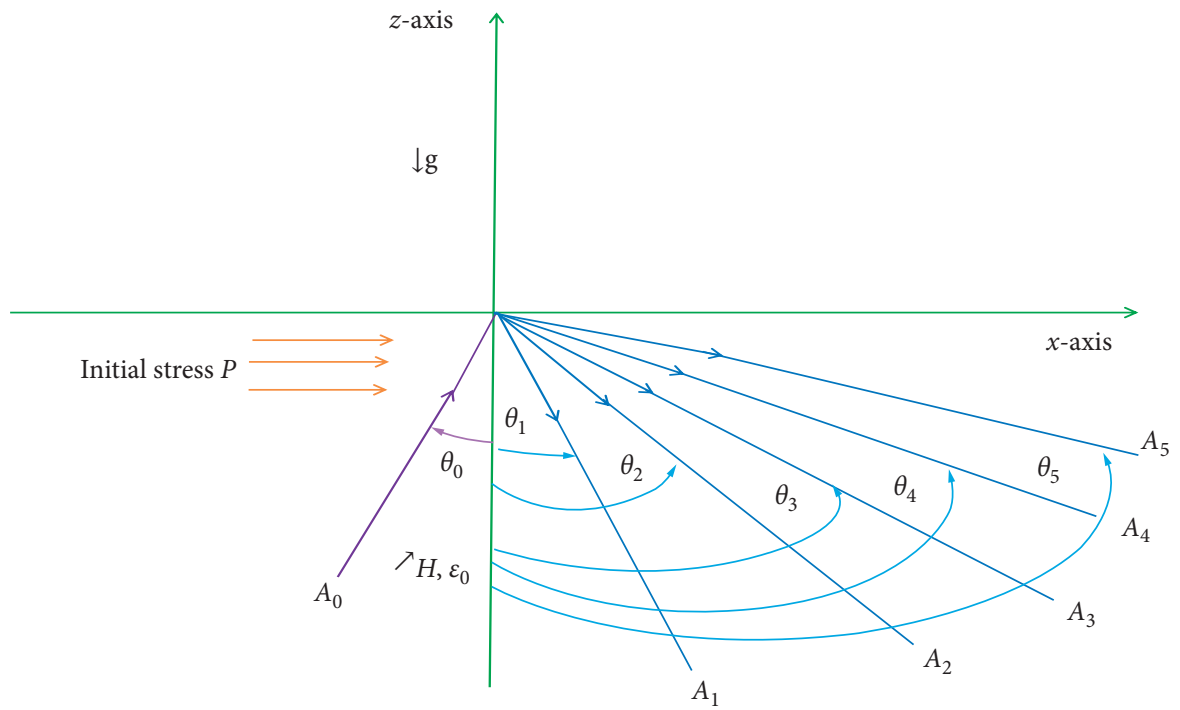

Figure 1: Geometry of the problem.

$$
\begin{aligned}
& B_{1}=-a_{1} \vartheta_{1}+\xi_{j}^{2}\left[a_{3}\left(\sin ^{2} \theta_{0}+\frac{\eta_{1}}{2} \sin 2 \theta_{0}\right)-a_{2}\left(\cos ^{2} \theta_{0}-\frac{\eta_{1}}{2} \sin 2 \theta_{0}+\frac{k_{j}}{\xi_{j}^{2}}\right)+\mu_{e} H_{0}^{2}\right], \\
& B_{2}=-\left(a_{4}+a_{5}\right) \frac{\xi_{1}^{2}}{2} \sin 2 \theta_{0}-a_{4} \xi_{1}^{2} \eta_{1} \cos ^{2} \theta_{1}+a_{5} \xi_{1}^{2} \eta_{1} \sin ^{2} \theta_{1}-a_{6} \xi_{1}, \\
& B_{3}=k_{1} \xi_{1} \cos \theta_{0}, \\
& B_{4}=\zeta_{1} \xi_{1} \cos \theta_{0}, \\
& B_{5}=-\vartheta_{1} \xi_{1} \cos \theta_{0} .
\end{aligned}
$$

\section{Numerical Results and Discussion}

The physical constants used are [3]

$$
\begin{aligned}
i & =\sqrt{-1}, \alpha_{0}=0.779 \times 10^{-4}, \lambda_{0}=0.5 \times 10^{11}, \lambda_{1}=0.5 \times 10^{11}, j=0.2 \times 10^{-15}, \rho=8954, C_{e}=383.1, \\
k & =386, T_{0}=293, \lambda=7.76 \times 10^{10}, \mu=3.86 \times 10^{10}, \gamma=0.779 \times 10^{-4}, \mathrm{~K}^{*}=2.97 \times 10^{13}, \mu_{0}=0.1, \\
\varepsilon_{0} & =0.1, \omega=0.24, \tau_{T}=0.002, \tau_{v}=0.001, \tau_{q}=0.005 .
\end{aligned}
$$

Figure 2 shows the variation of the amplitude of reflected $P_{1}$ wave $\left|Z_{1}\right|$, amplitude of reflected $P_{2}$ wave $\left|Z_{2}\right|$, amplitude of reflected $P_{3}$ wave $\left|Z_{3}\right|$, amplitude of reflected $P_{4}$ wave $\left|Z_{4}\right|$, and amplitude of reflected $P_{5}$ wave $\left|Z_{5}\right|$ with respect to the angle of incidence $\theta$ for different values of the initial stress. The amplitude of the reflected wave increases with increasing of the angle of incidence until it reaches a maximum value at $\theta$ about 90 . The amplitude of reflected $P_{2}$ wave $\left|Z_{2}\right|$, the amplitude of reflected $P_{3}$ wave $\left|Z_{3}\right|$, the amplitude of reflected $P_{4}$ wave $\left|Z_{4}\right|$, and the amplitude of reflected $P_{5}$ wave $\left|Z_{5}\right|$ each of them decrease with increasing of the angle of incidence until it becomes a minimum value at $\theta=90$. In addition, all lines begin to coincide when the horizontal angle of incidence $\theta$ increases to the maximum. These results obey physical reality for the behavior of reflection of $\mathrm{P}$ waves [3].

Figure 3 shows the variation of the amplitude of reflected $P_{1}$ wave $\left|Z_{1}\right|$, amplitude of reflected $P_{2}$ wave $\left|Z_{2}\right|$, amplitude of reflected $P_{3}$ wave $\left|Z_{3}\right|$, amplitude of reflected $P_{4}$ wave $\left|Z_{4}\right|$, and amplitude of reflected $P_{5}$ wave $\left|Z_{5}\right|$ with respect to the angle of incidence $\theta$ for different values of the gravitation. The amplitude of reflected $P_{1}$ wave $\left|Z_{1}\right|$, the amplitude of the reflected wave $\left|Z_{2}\right|$, and the amplitude of reflected $P_{5}$ wave $\left|Z_{5}\right|$ increases with increasing angle of incidence until it reaches a maximum value at $\theta$ about 90 . The amplitude of reflected $P_{3}$ wave $\left|Z_{3}\right|$ and the amplitude of reflected $P_{4}$ wave 


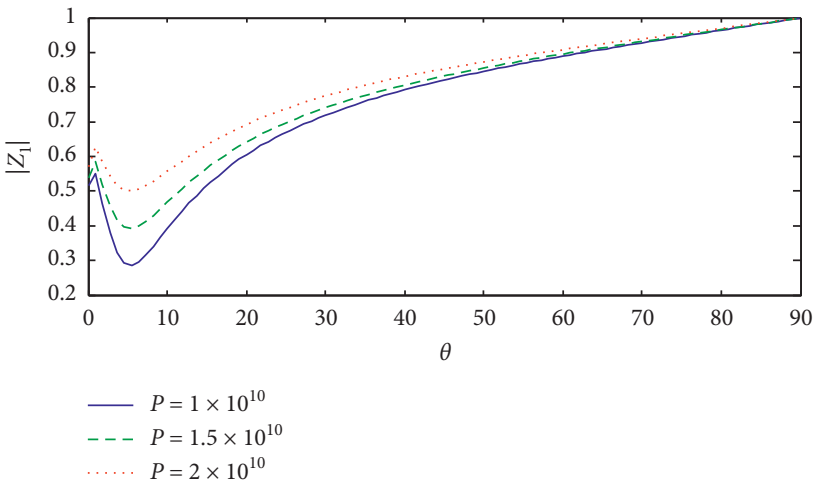

(a)

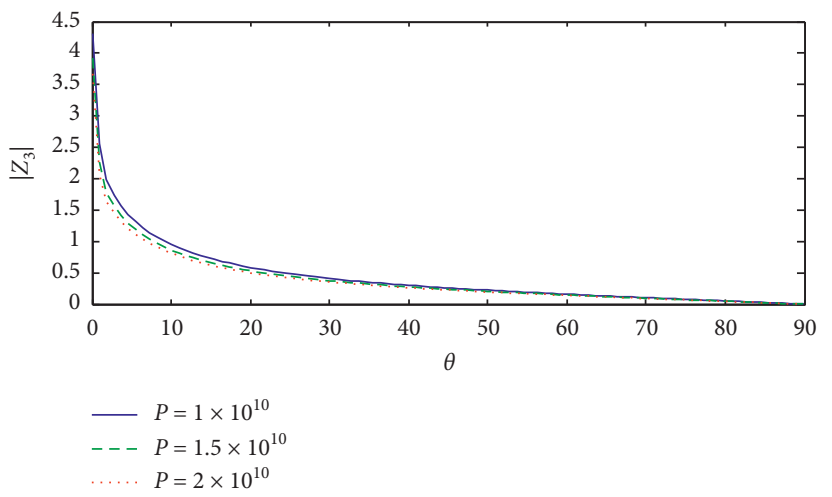

(c)

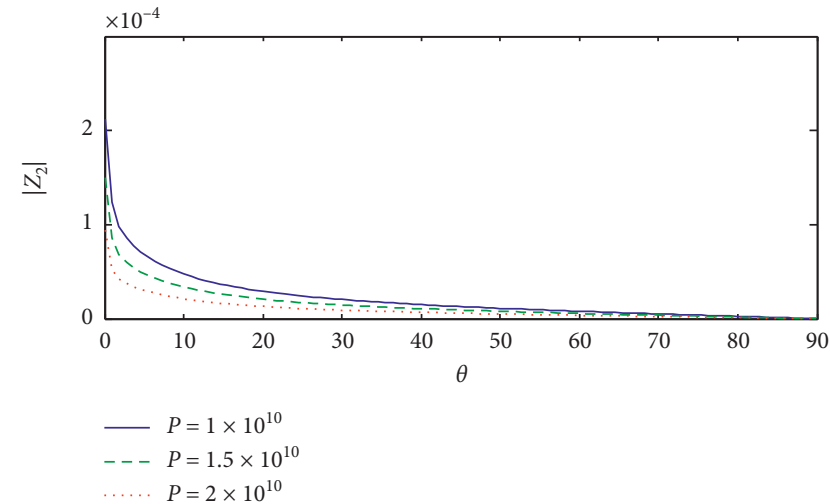

(b)

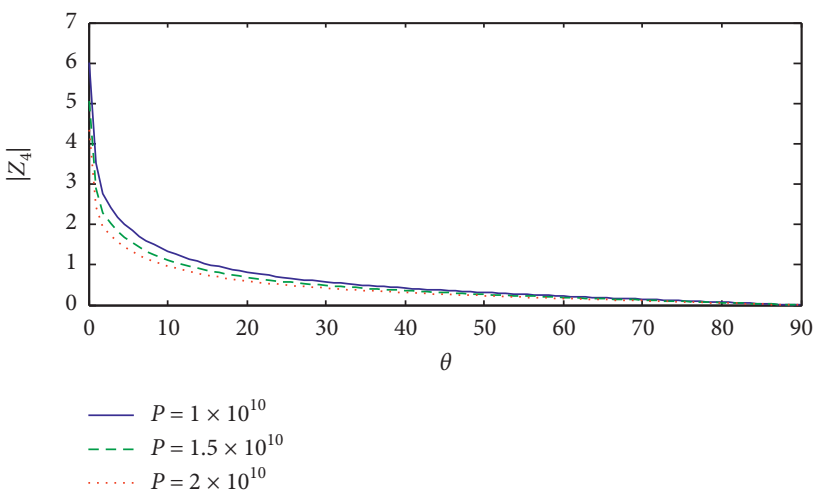

(d)

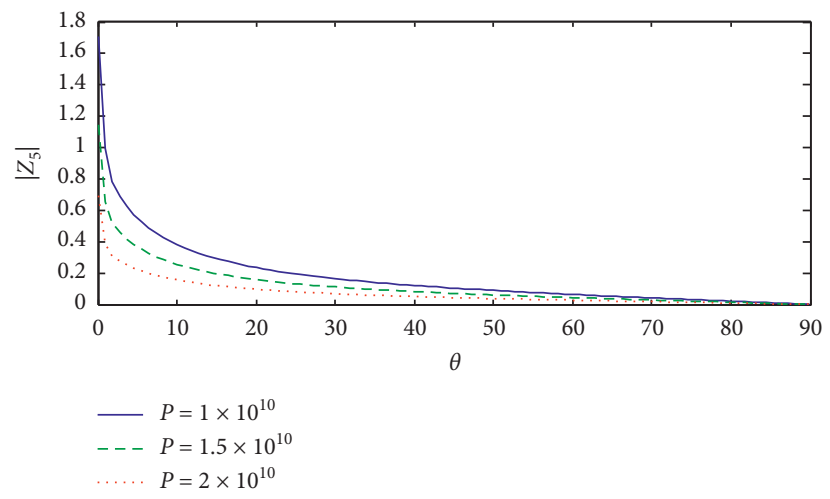

(e)

Figure 2: Variation of the amplitudes $\left|Z_{i}\right|(i=1,2, \ldots, 5)$ with the angle of incidence of $\mathrm{P}$ waves for variation of initial stress.

$\left|Z_{4}\right|$ decreases with increasing angle of incidence until it becomes a minimum value at $\theta=90$. It is noticed that the reflection of $\mathrm{P}$ waves is strongly affected by the presence of gravity [23].

Figures 4 and 5: show the variation of the amplitude of reflected $P_{1}$ wave $\left|Z_{1}\right|$, amplitude of reflected $P_{2}$ wave $\left|Z_{2}\right|$, amplitude of reflected $P_{3}$ wave $\left|Z_{3}\right|$, amplitude of reflected $P_{4}$ wave $\left|Z_{4}\right|$, and amplitude of reflected $P_{5}$ wave $\left|Z_{5}\right|$ with respect to the angle of incidence $\theta$ for different values of the electric field and magnetic field, respectively. The amplitude of reflected $P_{1}$ wave $\left|Z_{1}\right|$, amplitude of reflected $P_{2}$ wave $\left|Z_{2}\right|$, amplitude of reflected $P_{3}$ wave $\left|Z_{3}\right|$, amplitude of reflected $P_{4}$ wave $\left|Z_{4}\right|$, and amplitude of reflected $P_{5}$ wave $\left|Z_{5}\right|$ increase with increasing electric field and magnetic field. The electric field and magnetic field increase the magnitude of reflection of $\mathrm{P}$ waves. This is mainly due to the fact that the electric field and magnetic field correspond to a term signifying a positive force that tends to accelerate the charge carries [2].

Figure 6 shows the variation of the amplitude of reflected $P_{1}$ wave $\left|Z_{1}\right|$, amplitude of reflected $P_{2}$ wave $\left|Z_{2}\right|$, amplitude of reflected $P_{3}$ wave $\left|Z_{3}\right|$, amplitude of reflected $P_{4}$ wave $\left|Z_{4}\right|$, and amplitude of reflected $P_{5}$ wave $\left|Z_{5}\right|$ with respect to the angle of incidence $\theta$. The change of amplitude of reflected to wave appeared in this figure, where we notice $\left|Z_{3}\right|>\left|Z_{4}\right|>\left|Z_{5}\right|>\left|Z_{2}\right|>\left|Z_{1}\right|$ of the existence of gravity field 


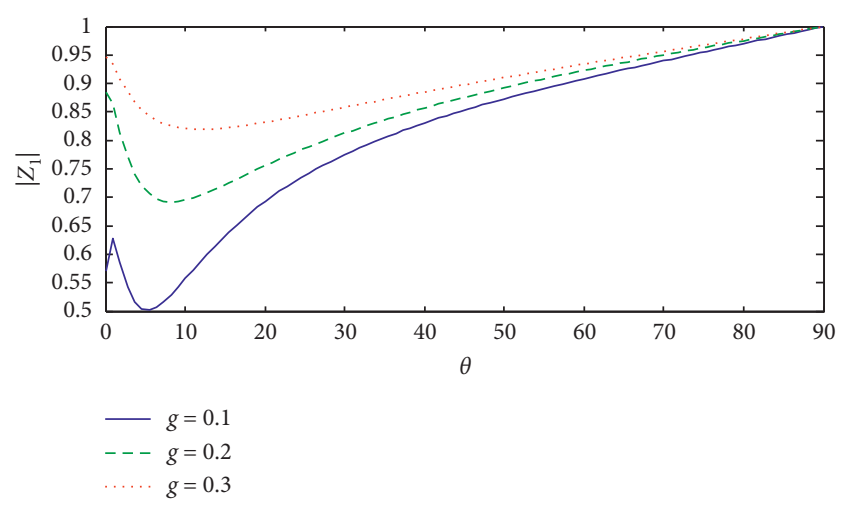

(a)

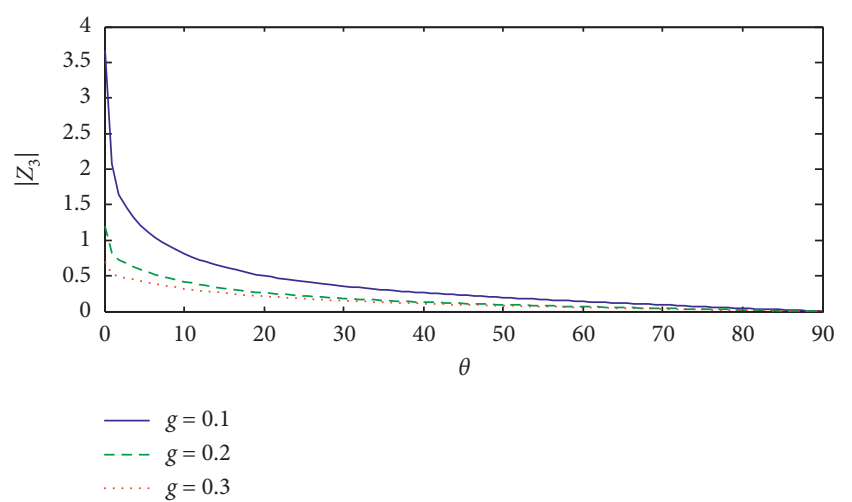

(c)

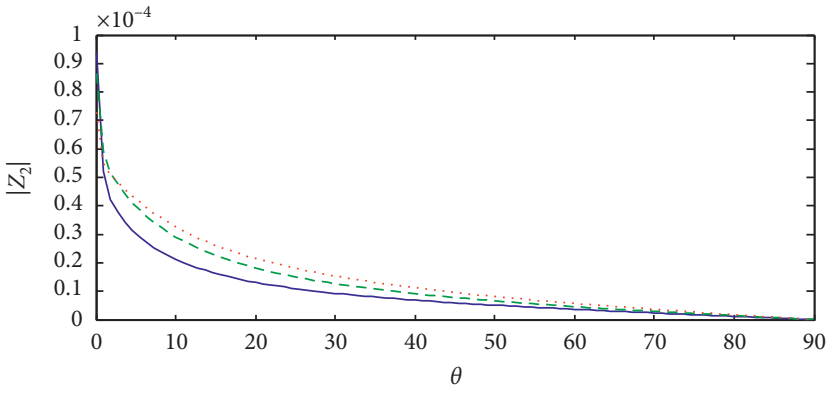

$$
\begin{aligned}
-g & =0.1 \\
--g & =0.2 \\
\cdots-g & =0.3
\end{aligned}
$$

(b)

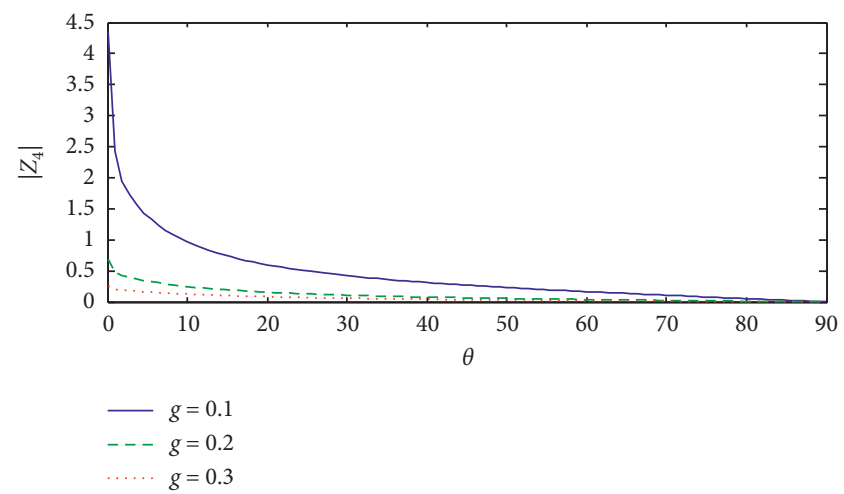

(d)

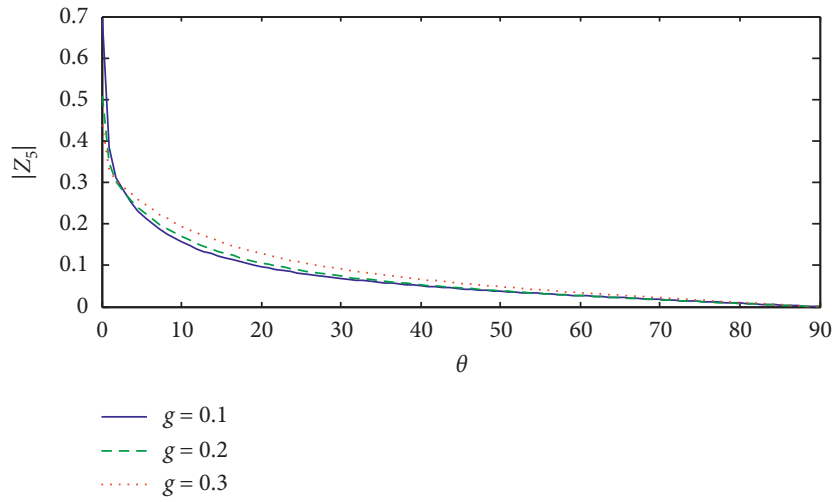

(e)

Figure 3: Variation of the amplitudes $\left|Z_{i}\right|(i=1,2, \ldots, 5)$ with the angle of incidence of $\mathrm{P}$ waves for variation of gravity field. 

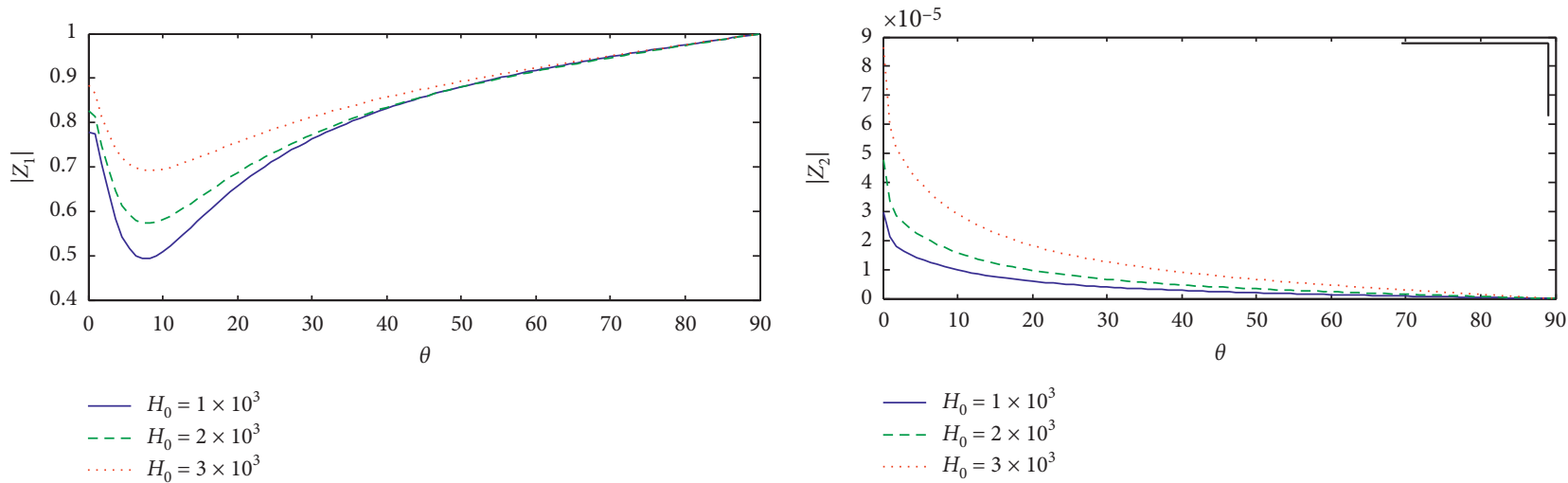

(a)
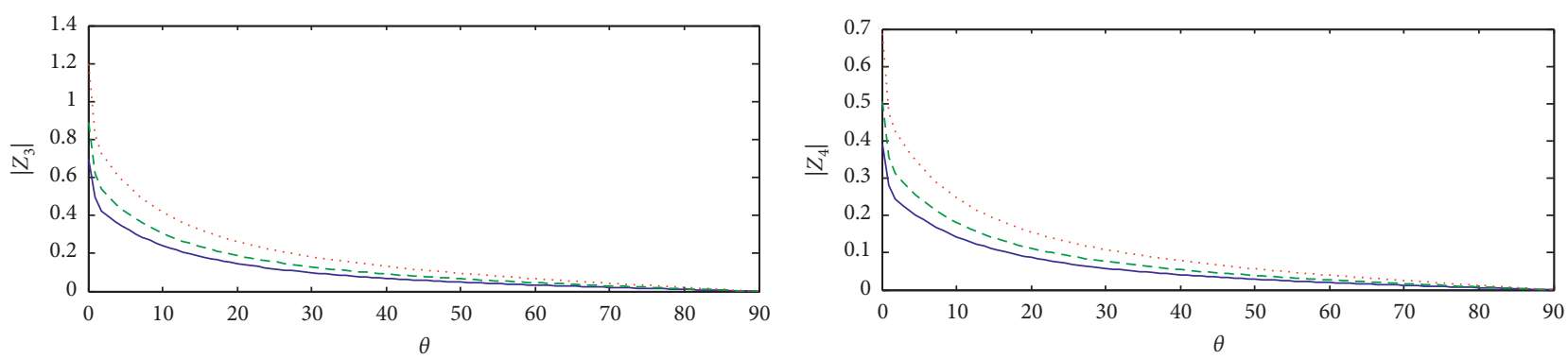

$$
\begin{aligned}
-H_{0} & =1 \times 10^{3} \\
---H_{0} & =2 \times 10^{3} \\
\text { …. } H_{0} & =3 \times 10^{3}
\end{aligned}
$$$$
\begin{aligned}
\text { - } H_{0} & =1 \times 10^{3} \\
\text { - - } H_{0} & =2 \times 10^{3} \\
\text { …. } H_{0} & =3 \times 10^{3}
\end{aligned}
$$

(c)

(d)

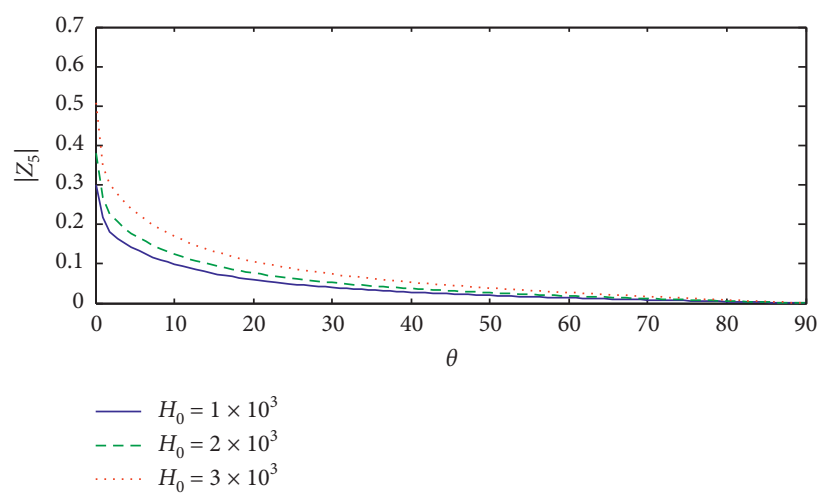

(e)

FIGURE 4: Variation of the amplitudes $\left|Z_{i}\right|(i=1,2, \ldots, 5)$ with the angle of incidence of $\mathrm{P}$ waves for variation of magnetic field. 

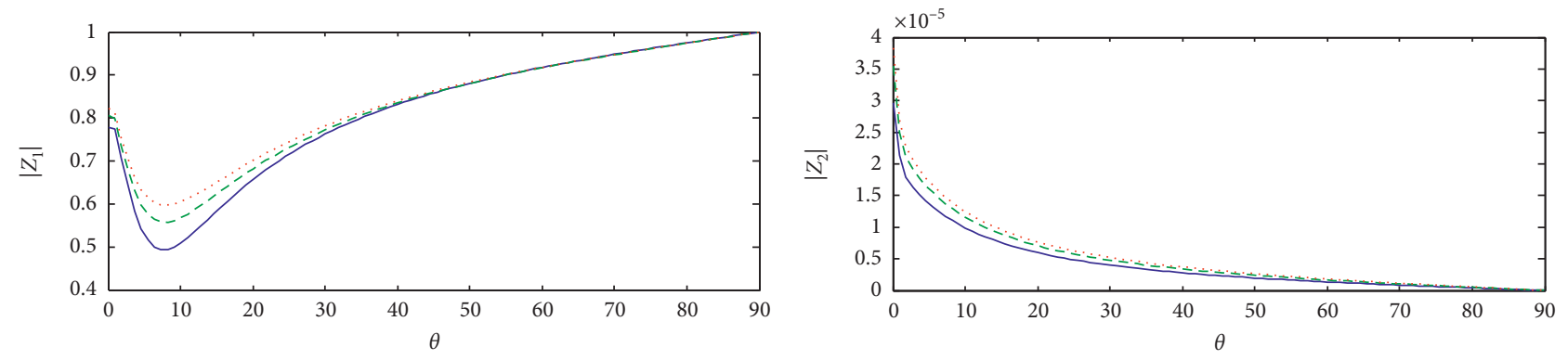

$$
\begin{aligned}
-\varepsilon_{0} & =0.1 \\
--- & \varepsilon_{0}=0.2 \\
\ldots \ldots & \varepsilon_{0}=0.25
\end{aligned}
$$

(a)

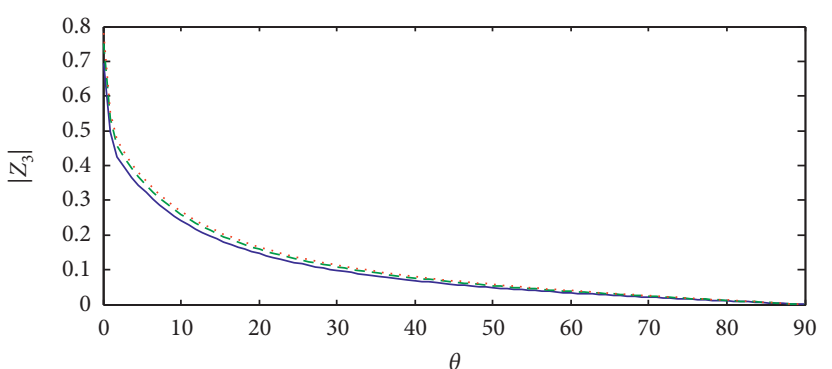

$$
\begin{aligned}
-\varepsilon_{0} & =0.1 \\
--- & \varepsilon_{0}=0.2 \\
\cdots \cdots & \varepsilon_{0}=0.25
\end{aligned}
$$

(b)

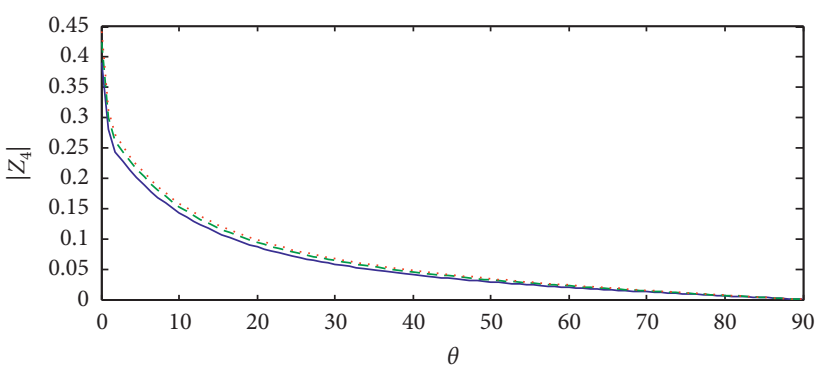

$$
\begin{aligned}
& -\varepsilon_{0}=0.1 \\
& ---\varepsilon_{0}=0.2 \\
& \ldots \ldots \varepsilon_{0}=0.25
\end{aligned}
$$

(c)

(d)

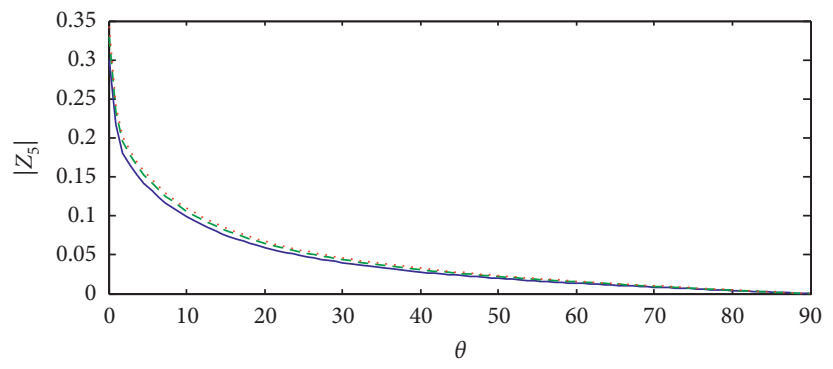

$$
\begin{aligned}
-\varepsilon_{0} & =0.1 \\
--- & \varepsilon_{0}=0.2 \\
\cdots \cdots & \varepsilon_{0}=0.25
\end{aligned}
$$

(e)

Figure 5: Variation of the amplitudes $\left|Z_{i}\right|(i=1,2, \ldots, 5)$ with the angle of incidence of $\mathrm{P}$ waves for variation of electric field.

gravitation, electric field, magnetic field, and initial stress. These figures are very important to study the dependence of these physical quantities on the vertical amplitude of reflected of distance. The curves obtained are highly depending on the vertical distance from origin, and all the physical quantities satisfy boundary condition and are moving in $\mathrm{P}$ waves [24]. 


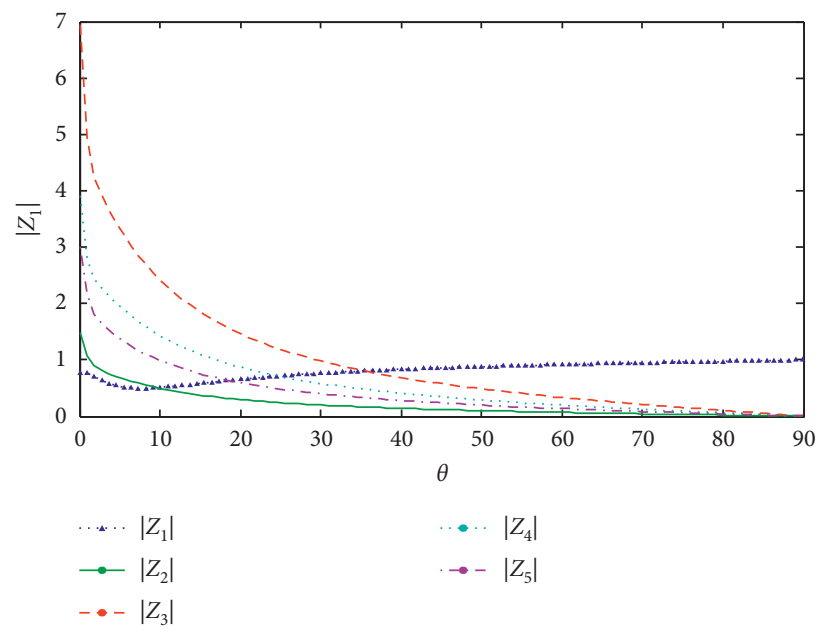

Figure 6: Variation of the amplitudes $\left|Z_{i}\right|(i=1,2, \ldots, 5)$ with the angle of incidence of $\mathrm{P}$ waves: $P=10^{10}, \varepsilon_{0}=0.1, g=0.1$, and $H_{0}=10^{3}$.

\section{Conclusion}

We obtain the following conclusions based on the above analysis:

(1) The reflected $P$ waves and amplitude of the reflected $\mathrm{P}$ waves depend on the angle of incidence, rotation, initial stress, gravity field, and magnetic field, and the nature of this dependence is different for different reflected $\mathrm{P}$ waves.

(2) The rotation, initial stress, gravity field, and electromagnetic field play a significant role, and the effects have the inverse trend for the reflected $\mathrm{P}$ waves and amplitude of the reflected $\mathrm{P}$ waves.

(3) The rotation, initial stress, gravity field, and electromagnetic field have a strong effect on the reflected $\mathrm{P}$ waves and amplitude of the reflected $\mathrm{P}$ waves.

(4) The result provides a motivation to investigate the reflection of $\mathrm{P}$ waves from thermo-magneto-microstretch materials as a new class of applications thermoelectric solids. The results presented in this paper should prove to be useful for researchers in material science, designers of new materials, microsystem technologies, physicists, as well as for those working on the development of thermo-electro-magneto-microstretch and in practical situations as in geophysics, optics, acoustics, and geomagnetic and oil prospecting.

(5) The equations were solved by using the wave function assumption which is the basis of the normal mode method and the features of this system. We can find the amplitude of the primary wave under the influence of different variables, whether gravity, primary gain, and electric and magnetic fields, by varying relaxation time.

It is observed that the amplitudes of reflected $\mathrm{P}$ waves change in the presence of rotation, initial stress, gravity field, and magnetic field. Hence, the presence of rotation, initial stress, gravity field, and electromagnetic field significantly affected the reflection phenomena.

\section{Nomenclature}

$\vec{B}: \quad$ Magnetic induction vector

$C_{e}: \quad$ Specific heat per unit mass

$\vec{E}$ : $\quad$ Electric intensity vector

$e_{i j}: \quad$ Strain tensor

$\vec{F}_{i}: \quad$ Lorentz's body force vector

g: $\quad$ Gravity field

$\vec{H}$ : $\quad$ Magnetic field vector

$\vec{h}$ : $\quad$ Perturbed magnetic field vector

$\vec{H}_{0}$ : $\quad$ Primary constant magnetic field vector

$i=\sqrt{-1}, j:$ Microinertia moment

$\vec{J}: \quad$ Electric current density vector

$\mathrm{K}, K^{*}$ : Thermal conduction coefficients

$m_{i j}: \quad$ Coupled stress tensor

$P: \quad$ Initial stress

$t$ : Time

$T_{0}$ : The reference temperature

T: $\quad$ The temperature distribution

u: $\quad$ Displacement vector

$\alpha_{0}, \lambda_{0}, \lambda_{1}:$ Microstretch constants

$\alpha_{t}$ : $\quad$ Coefficient of linear thermal expansion

$\delta_{i j}: \quad$ Kronecker delta

$\varepsilon_{0}$ : $\quad$ Electric permeability

$\varphi$ : $\quad$ Rotation vector

$\varphi^{*}: \quad$ Scalar microstretch

$k, \alpha, \beta, \gamma:$ Micropolar constants

$\lambda, \mu: \quad$ Lame' constants

$\mu_{0}: \quad$ Magnetic permeability

$v=(\omega / \xi)$ : Velocity of the coupled waves

$\rho: \quad$ Mass density

$\sigma_{i j}: \quad$ Stress tensor

$\tau_{i j}$ : $\quad$ Maxwell's stress tensor

$\tau_{q}: \quad$ The phase-lag of the heat flux

$\tau_{T}$ : The phase-lag of temperature gradient

$\tau_{v}: \quad$ Thermal displacement phase lag

$\omega: \quad$ Complex frequency

$\xi: \quad$ Wave number

$\widehat{\gamma}: \quad(3 \lambda+2 \mu+k) \alpha_{t_{1}}$

$\widehat{\gamma}_{1}: \quad(3 \lambda+2 \mu+k) \alpha_{t_{2}}$. 


\section{Appendix}

The coefficients of Eq. (45)

$$
\begin{aligned}
& A=-\frac{C_{1}^{2}}{c_{0}^{2}} L \\
& B=\frac{C_{1}^{2}}{c_{0}^{2}}\left(p^{*} L \zeta_{6}-\zeta_{2} \varepsilon_{1} \omega^{2} \tau_{q}^{*}+L \omega^{2} \zeta_{5}+\zeta_{0} \omega^{2} L+L S-\omega^{2} \tau_{q}^{*}\right)-\frac{C_{2}^{2}}{\omega^{* 2}} L+\omega^{2} L+\zeta_{3} L \frac{C_{3}^{2}}{\omega^{* 2}}, \\
& C=-a_{0} \varepsilon_{2} \omega^{2} \tau_{q}^{*}-\frac{C_{2}^{2}}{\omega^{* 2}} \omega^{2} \tau_{q}^{*}+\omega^{4} \tau_{q}^{*}+\frac{C_{1}^{2}}{c_{0}^{4}} \omega^{4} \tau_{q}^{*} \zeta_{5}+\frac{C_{2}^{2}}{\omega^{* 2}} L \omega^{2} \zeta_{5}-\omega^{4} L \zeta_{6}+\frac{C_{1}^{2}}{c_{0}^{2}} \omega^{4} \tau_{q}^{*} \zeta_{0}+\frac{C_{2}^{2}}{\omega^{* 2}} L \zeta_{0} \omega^{2} \\
& -\omega^{4} L \zeta_{0}-\frac{C_{1}^{2}}{c_{0}^{2}} L \zeta_{0} \zeta_{5} \omega^{4}+\frac{C_{1}^{2}}{c_{0}^{2}} \omega^{2} \tau_{q}^{*} S+\frac{C_{2}^{2}}{\omega^{* 2}} L S-C_{1}^{2} L S \omega^{2} \zeta_{5}-\omega^{2} L S-\frac{C_{1}^{2}}{c_{0}^{2}} L S \zeta_{0} \omega^{2}-\zeta_{1} \zeta_{4} \sin ^{2} \vartheta \frac{C_{1}^{2}}{c_{0}^{2}} L \\
& +p^{*} \frac{C_{1}^{2}}{c_{0}^{2}} \omega^{2} \tau_{q}^{*} \zeta_{6}+p^{*} \frac{C_{2}^{2}}{\omega^{* 2}} L \zeta_{6}+p^{*} \frac{C_{2}^{2}}{\omega^{* 2}} \omega^{2} \tau_{q}^{*} \zeta_{6}-p^{*} \omega^{2} L \zeta_{6}-\zeta_{6} \zeta_{0} \omega^{2} \frac{C_{1}^{2}}{c_{0}^{2}} L p^{*}+\zeta_{2} \frac{C_{3}^{2}}{\omega^{* 2} \varepsilon_{2} \omega^{2} \tau_{q}^{*}} \\
& -\zeta_{2} \varepsilon_{1} \omega^{2} \tau_{q}^{*} \frac{C_{2}^{2}}{\omega^{* 2}}+\zeta_{2} \varepsilon_{1} \omega^{4} \tau_{q}^{*}-\zeta_{3} L S \frac{C_{3}^{2}}{\omega^{* 2}}-\zeta_{3} \zeta_{5} L \omega^{2} \frac{C_{3}^{2}}{\omega^{* 2}}-\zeta_{3} \zeta_{6} P^{*} L \frac{C_{3}^{2}}{\omega^{* 2}}+\zeta_{2} \varepsilon_{1} \omega^{2} \tau_{q}^{*} S \frac{C_{1}^{2}}{c_{0}^{2}} \\
& +\zeta_{2} \zeta_{5} \varepsilon_{1} \omega^{4} \tau_{q}^{*} \frac{C_{1}^{2}}{c_{0}^{2}}-\zeta_{2} \zeta_{6} p^{*} \varepsilon_{1} \omega^{2} \tau_{q}^{*} \frac{C_{1}^{2}}{c_{0}^{2}}+\zeta_{3} \omega^{2} \tau_{q}^{*} \frac{C_{3}^{2}}{\omega^{* 2}}+\zeta_{3} a_{0} \varepsilon_{1} \omega^{2} \tau_{q}^{*} \\
& D=\omega^{4} \zeta_{5} a_{0} \varepsilon_{2} \tau_{q}^{*}+\frac{C_{2}^{2}}{\omega^{* 2}} \omega^{4} \tau_{q}^{*} \zeta_{5}-\omega^{6} \tau_{q}^{*} \zeta_{5}-a_{0} \varepsilon_{2} \omega^{4} \zeta_{0} \tau_{q}^{*}+\frac{C_{2}^{2}}{\omega^{* 2}} \omega^{4} \zeta_{0} \tau_{q}^{*}-\omega^{6} \zeta_{0} \tau_{q}^{*}+\frac{C_{2}^{2}}{\omega^{* 2}} \omega^{2} \tau_{q}^{*} S \\
& -\frac{C_{1}^{2}}{c_{0}^{2}} \omega^{6} \tau_{q}^{*} \zeta_{0} \zeta_{5}-\frac{C_{2}^{2}}{\omega^{* 2}} L \zeta_{0} \zeta_{5} \omega^{4}+\omega^{6} L \zeta_{0} \zeta_{5}+a_{0} \varepsilon_{2} \omega^{2} S \tau_{q}^{*}-\omega^{4} \tau_{q}^{*} S-S \zeta_{0} \omega^{2} \frac{C_{2}^{2}}{\omega^{* 2}} L+\omega^{4} L S \zeta_{0} \\
& -\frac{C_{1}^{2}}{c_{0}^{2}} \omega^{4} \tau_{q}^{*} S \zeta_{5}-\frac{C_{2}^{2}}{\omega^{* 2}} L S \omega^{2} \zeta_{5}+\omega^{4} L S \zeta_{5}-\frac{C_{1}^{2}}{c_{0}^{2}} \omega^{4} \tau_{q}^{*} S \zeta_{0}-\zeta_{1} \zeta_{4} \sin ^{2} \vartheta \frac{C_{1}^{2}}{c_{0}^{2}} \omega^{2} \tau_{q}^{*}-\zeta_{1} \zeta_{4} \sin ^{2} \vartheta \frac{C_{2}^{2}}{\omega^{* 2}} L \\
& +S \zeta_{0} \zeta_{5}{ }^{4} \frac{C_{1}^{2}}{c_{0}^{2}} L+\zeta_{1} \zeta_{4} \sin ^{2} 9 \frac{C_{1}^{2}}{c_{0}^{2}} L S-\zeta_{0} \zeta_{6} p^{*} \omega^{4} \tau_{q}^{*} c_{1}^{2}-\zeta_{0}^{2} \zeta_{6} p^{*} \omega^{2} \frac{C_{2}^{2}}{\omega^{* 2}} L-p^{*} \omega^{4} \tau_{q}^{*} \zeta_{6} \\
& +\zeta_{1} \zeta_{4} \sin ^{2} \vartheta \omega^{2} L+p^{*} a_{0} \varepsilon_{2} \omega^{2} \tau_{q}^{*} \zeta_{6}+\zeta_{2} S \frac{C_{2}^{2}}{\omega^{* 2}} \varepsilon_{1} \tau_{q}^{*} \omega^{2}-\zeta_{2} S \varepsilon_{1} \tau_{q}^{*} \omega^{4}-\zeta_{2} \zeta_{5} \frac{C_{3}^{2}}{\omega^{* 2}} \varepsilon_{2} \tau_{q}^{*} \omega^{4}-\zeta_{2} \zeta_{5} \varepsilon_{1} \tau_{q}^{*} \omega^{6} \\
& +\zeta_{0} \zeta_{6} p^{*} \omega^{4} L-\zeta_{2} S \frac{C_{3}^{2}}{\omega^{* 2}} \varepsilon_{2} \tau_{q}^{*} \omega^{2}-\zeta_{3} \zeta_{5} \omega^{4} \tau_{q}^{*} a_{0} \varepsilon_{1}+\zeta_{3} \zeta_{5} \omega^{2} L S \frac{C_{3}^{2}}{\omega^{* 2}}-\zeta_{3} \zeta_{6} \omega^{2} \tau_{q}^{*} a_{0} \varepsilon_{1}-\zeta_{3} \zeta_{5} \omega^{4} \tau_{q}^{*} \frac{C_{3}^{2}}{\omega^{* 2}} \\
& +\zeta_{2} \zeta_{5} \frac{C_{2}^{2}}{\omega^{* 2}} \varepsilon_{1} \tau_{q}^{*} \omega^{4}-\zeta_{2} \zeta_{5} \varepsilon_{1} \tau_{q}^{*} \omega^{4} S \frac{C_{1}^{2}}{c_{0}^{2}}+\zeta_{2} \zeta_{6} p^{*} \omega^{2} \tau_{q}^{*} \varepsilon_{2} \frac{C_{3}^{2}}{\omega^{* 2}}-\zeta_{3} S \omega^{2} \tau_{q}^{*} \frac{C_{3}^{2}}{\omega^{* 2}}-\zeta_{3} S a_{0} \varepsilon_{1} \tau_{q}^{*} \omega^{2}
\end{aligned}
$$




$$
\begin{aligned}
& -\zeta_{2} \zeta_{6} p^{*} \omega^{2} \tau_{q}^{*} \varepsilon_{1} \frac{C_{2}^{2}}{\omega^{* 2}}+\zeta_{2} \zeta_{6} p^{*} \omega^{4} \tau_{q}^{*} \varepsilon_{1}, \\
& E=a_{0} \varepsilon_{2} \omega^{6} \zeta_{0} \zeta_{5} \tau_{q}^{*}-\frac{C_{2}^{2}}{\omega^{* 2}} \omega^{6} \tau_{q}^{*} \zeta_{0} \zeta_{5}+\omega^{8} \zeta_{0} \zeta_{5} \tau_{q}^{*}-S \omega^{4} \zeta_{5} a_{0} \varepsilon_{2} \tau_{q}^{*}-\frac{C_{2}^{2}}{\omega^{* 2}} \omega^{4} \tau_{q}^{*} \zeta_{5} S-\zeta_{3} \zeta_{6} p^{*} \omega^{2} \tau_{q}^{*} \frac{C_{3}^{2}}{\omega^{* 2}} \\
& +\omega^{6} \tau_{q}^{*} \zeta_{5} S-\omega^{4} \tau_{q}^{*} \zeta_{0} S a_{0} \varepsilon_{2}-\frac{C_{2}^{2}}{\omega^{* 2}} \omega^{4} \tau_{q}^{*} S \zeta_{0}+\omega^{6} \tau_{q}^{*} \zeta_{0} S+\frac{C_{1}^{2}}{c_{0}^{2}} \omega^{6} \tau_{q}^{*} S \zeta_{0} \zeta_{5}-S \zeta_{0} \zeta_{5} \omega^{6} L \\
& +\frac{C_{2}^{2}}{\omega^{* 2}} L S \zeta_{0} \zeta_{5} \omega^{4}+\sin ^{2} \vartheta \zeta_{1} \zeta_{4} S \frac{C_{1}^{2}}{c_{0}^{2}} \omega^{2} \tau_{q}^{*}+\zeta_{1} \zeta_{4} \sin ^{2} \vartheta \frac{C_{2}^{2}}{\omega^{* 2}} L S+\zeta_{1} \zeta_{4} \sin ^{2} \vartheta \omega^{4} \tau_{q}^{*}+\zeta_{2} \zeta_{5} \omega^{6} S \varepsilon_{1} \tau_{q}^{*} \\
& -\zeta_{1} \zeta_{4} \sin ^{2} \vartheta \omega^{2} L S-\zeta_{1} \zeta_{4} \sin ^{2} \vartheta \omega^{2} a_{0} \varepsilon_{2} \tau_{q}^{*}-\zeta_{1} \zeta_{4} \sin ^{2} \vartheta \frac{C_{2}^{2}}{\omega^{* 2}} \omega^{2} \tau_{q}^{*}+\zeta_{2} \zeta_{5} \omega^{4} S \frac{C_{3}^{2}}{\omega^{* 2}} \varepsilon_{2} \tau_{q}^{*}-\zeta_{2} \zeta_{5} \omega^{4} S \frac{C_{2}^{2}}{\omega^{* 2}} \varepsilon_{1} \tau_{q}^{*} \\
& +\zeta_{0} \zeta_{6} \omega^{6} p^{*} \tau_{q}^{*}-\zeta_{0} \zeta_{6} p^{*} a_{0} \varepsilon_{2} \omega^{4} \tau_{q}^{*}-\zeta_{0} \zeta_{6} p^{*} \omega^{4} \tau_{q}^{*} \frac{C_{2}^{2}}{\omega^{* 2}}+\zeta_{3} \zeta_{5} \omega^{4} S \varepsilon_{1} \tau_{q}^{*} a_{0}+\zeta_{3} \zeta_{5} \omega^{4} S \frac{C_{3}^{2}}{\omega^{* 2}} \tau_{q}^{*} \text {, } \\
& F=\tau_{q}^{*} S \omega^{2}\left(\zeta_{0} \zeta_{5} \omega^{4} a_{0} \varepsilon_{2}+\zeta_{0} \zeta_{5} \omega^{4} \frac{C_{2}^{2}}{\omega^{* 2}}+\zeta_{1} \zeta_{4} \sin ^{2} \vartheta a_{0} \varepsilon_{2}+\zeta_{1} \zeta_{4} \sin ^{2} \vartheta \frac{C_{2}^{2}}{\omega^{* 2}}-\zeta_{1} \zeta_{4} \sin ^{2} \vartheta \omega^{2}\right) \\
& S=\frac{-j \rho c_{0}^{2} \omega^{2}}{\widehat{\gamma}}+\frac{2 k c_{0}^{2}}{\tilde{\gamma} \omega^{* 2}} \\
& L=-C_{k}-i \omega C_{v}+\omega^{2} C_{T} \text {. }
\end{aligned}
$$

\section{The amplitudes of Eq. (46)}

$$
\begin{aligned}
& \eta_{j}=\frac{-l \xi_{j} \zeta_{4} \sin \theta_{j} M_{2}}{\left(\xi_{j}^{2}-\omega^{2} \zeta_{5}\right) M_{2}-\rho^{*} \xi_{j}^{2} \zeta_{6}}, \\
& \kappa_{j}=\frac{i \rho^{*} \xi_{j}^{3} \zeta_{4} \sin \theta_{j}}{\left(\xi_{j}^{2}-\omega^{2} \zeta_{5}\right) M_{2}-\rho^{*} \xi_{j}^{2} \zeta_{6}}, \\
& \chi_{j}=\frac{-\varepsilon_{2} \omega^{2} \tau_{q}^{*} \xi_{j}^{2}\left(C_{3}^{2} / \omega^{* 2}\right)-\varepsilon_{1} \omega^{2} \tau_{q}^{*} M_{1} \xi_{j}^{2}}{a_{0} \varepsilon_{2} \omega^{2} \tau_{q}^{*}-\left[L \xi_{j}^{2}+\omega^{2} \tau_{q}^{*}\right] M_{1}}, \\
& \vartheta_{j}=\frac{a_{0} \varepsilon_{1} \omega^{2} \tau_{q}^{*} \xi_{j}^{2}+\left(C_{3}^{2} / \omega^{* 2}\right) \xi_{j}^{2}\left[L \xi_{j}^{2}+\omega^{2} \tau_{q}^{*}\right]}{a_{0} \varepsilon_{2} \omega^{2} \tau_{q}^{*}-\left[L \xi_{j}^{2}+\omega^{2} \tau_{q}^{*}\right] M_{1}}, \\
& M_{1}=\left[-\frac{\left.C_{1}^{2} \xi_{j}^{2}-\frac{C_{2}^{2}}{c_{0}^{2}}+\omega^{2}\right],}{c_{2}}=\xi_{j}^{2}+S .\right.
\end{aligned}
$$

\section{Data Availability}

The data used to support the findings of this study are available from the authors upon request.

\section{Conflicts of Interest}

The authors declare that they have no conflicts of interest.

\section{Acknowledgments}

The authors would like to acknowledge the financial support of Taif University Researchers Supporting Project (TURSP2020/164), Taif University, Taif, Saudi Arabia.

\section{References}

[1] M. N. M. Allam, S. Z. Rida, S. M. Abo-Dahab, R. A. Mohamed, and A. A. Kilany, "GL model on reflection of $\mathrm{P}$ and SV waves from the free surface of thermoelastic diffusion solid under influence of the electromagnetic field and initial stress," Journal of Thermal Stresses, vol. 37, no. 4, pp. 471-487, 2014.

[2] S. M. Abo-Dahab and A. Kilicman, "On reflection and transmission of $\mathrm{p}$ - and SV-waves phenomena at the interface between solid-liquid media with magnetic field and two thermal relaxation times," Journal of Thermal Stresses, vol. 38, no. 5, pp. 447-467, 2015.

[3] M. I. A. Othman and Y. Song, "Reflection of plane waves from an elastic solid half-space under hydrostatic initial stress without energy dissipation," International Journal of Solids and Structures, vol. 44, no. 17, pp. 5651-5664, 2007.

[4] A. M. Abd-Alla, S. M. Abo-Dahab, and A. A. Kilany, "SVwaves incidence at interface between solid-liquid media under electromagnetic field and initial stress in the context of three thermoelastic theories," Journal of Thermal Stresses, vol. 39, no. 8, pp. 960-976, 2016.

[5] A. E. Aboueregal and S. M. Abo-Dahab, "Dual-Phase-model on magneto-thermoelasticity infinite non-homogeneous solid having a spherical cavity," Journal of Thermal Stresses, vol. 35, no. 9, pp. 820-841, 2012.

[6] Z.-D. Zhou, F.-P. Yang, and Z.-B. Kuang, "Reflection and transmission of plane waves at the interface of pyroelectric bimaterials," Journal of Sound and Vibration, vol. 331, no. 15, pp. 3558-3566, 2012. 
[7] B. Singh, "Reflection of $\mathrm{P}$ and SV waves from free surface of an elastic solid with generalized thermodiffusion," Journal of Earth System Science, vol. 114, no. 2, pp. 159-168, 2005.

[8] A. N. Sinha and S. B. Sinha, "Reflection of thermoelastic waves at a solid half-space with thermal relaxation," Journal of Physics of the Earth, vol. 22, no. 2, pp. 237-244, 1974.

[9] S. B. Sinha and K. A. Elsibai, "Reflection and refraction of thermoelastic waves at an interface of two semi-infinite media with two relaxation times," Journal of Thermal Stresses, vol. 20, no. 2, pp. 129-145, 1997.

[10] S. B. Sinha and K. A. Elsibai, "Reflection of thermoelastic waves at a solid half-space with two relaxation times," Journal of Thermal Stresses, vol. 19, no. 8, pp. 749-762, 1996.

[11] Y. C. Angel and J. D. Achenbach, "Reflection and transmission of elastic waves by a periodic array of cracks," Journal of Applied Mechanics, vol. 52, no. 1, pp. 33-41, 1985.

[12] A. A. Kilany, S. M. Abo-Dahab, A. M. Abd-Alla, and A. N. Abd-alla, "Photothermal and void effect of a semiconductor rotational medium based on Lord-Shulman theory," Mechanics Based Design of Structures and Machines, pp. 1-14, 2020.

[13] R. Quintanilla and R. Racke, "A note on stability in threephase-lag heat conduction," International Journal of Heat and Mass Transfer, vol. 51, no. 1-2, pp. 24-29, 2008.

[14] S. Kothari, R. Kumar, and S. Mukhopadhyay, "On the fundamental solutions of generalized thermoelasticity with three phase-lags," Journal of Thermal Stresses, vol. 33, no. 11, pp. 1035-1048, 2010.

[15] S. Banik and M. Kanoria, "Generalized thermoelastic interaction in a functionally graded isotropic unbounded medium due to varying heat source with three-phase-lag effect," Mathematics and Mechanics of Solids, vol. 18, no. 3, pp. 231-245, 2012.

[16] Y. Chou and R.-J. Yang, "Two-dimensional dual-phase-lag thermal behavior in single-/multi-layer structures using CESE method," International Journal of Heat and Mass Transfer, vol. 52, no. 1-2, pp. 239-249, 2009.

[17] K.-C. Liu, "Numerical analysis of dual-phase-lag heat transfer in a layered cylinder with nonlinear interface boundary conditions," Computer Physics Communications, vol. 177, no. 3, pp. 307-314, 2007.

[18] S. Marzougui, M. Bouabid, F. Mebarek-Oudina, N. AbuHamdeh, M. Magherbi, and K. Ramesh, "A computational analysis of heat transport irreversibility phenomenon in a magnetized porous channel," International Journal of $\mathrm{Nu}$ merical Methods for Heat \& Fluid Flow, 2020.

[19] S. M. Abo-Dahab, M. A. Abdelhafez, F. Mebarek-Oudina, and S. M. Bilal, "MHD Casson nanofluid flow over nonlinearly heated porous medium in presence of extending surface effect with suction/injection," Indian Journal of Physics, 2021.

[20] A. Zaim, A. Aissa, F. Mebarek-Oudina et al., "Galerkin finite element analysis of magneto-hydrodynamic natural convection of $\mathrm{Cu}$-water nanoliquid in a baffled U-shaped enclosure," Propulsion and Power Research, vol. 9, no. 4, pp. 383-393, 2020.

[21] F. Mebarek-Oudina, R. Bessaih, B. Mahanthesh, A. J. Chamkha, and J. Raza, "Magneto-thermal-convection stability in an inclined cylindrical annulus filled with a molten metal," International Journal of Numerical Methods for Heat \& Fluid Flow, vol. 31, no. 4, pp. 1172-1189, 2020.

[22] M. A. Ezzat and M. I. Othman, "Electromagneto-thermoelastic plane waves with two relaxation times in a medium of perfect conductivity," International Journal of Engineering Science, vol. 38, no. 1, pp. 107-120, 2000.
[23] K. Lotfy and M. E. Gabr, "Gravity effect on the dual-phase-lag model for plane waves of a fiber-reinforced micropolar thermoelastic medium in contact with Newtonian inviscid fluid," Applied Mathematics \& Information Sciences, vol. 12, no. 2, pp. 369-378, 2018.

[24] S. M. Abo-Dahab, A. M. Abd-Alla, A. A. Kilany, and M. Elsagheer, "Effect of rotation and gravity on the reflection of $\mathrm{P}$-waves from thermo-magneto-microstretch medium in the context of three phase lag model with initial stress," Microsystem Technologies, vol. 24, no. 8, pp. 3357-3369, 2018. 